\title{
Impact of balloon guide catheter on technical and clinical outcomes: a systematic review and meta- analysis
}

\author{
Waleed Brinjikji, ${ }^{1,2,3}$ Robert M Starke, ${ }^{4,5}$ M Hassan Murad, ${ }^{6}$ David Fiorella, ${ }^{7}$ \\ Vitor M Pereira, ${ }^{8}$ Mayank Goyal, $^{9}$ David F Kallmes ${ }^{1,2}$
}

\begin{abstract}
${ }^{1}$ Department of Radiology, Mayo Clinic, Rochester,

Minnesota, USA

${ }^{2}$ Department of Neurosurgery,

Mayo Clinic, Rochester,

Minnesota, USA

${ }^{3}$ Department of Neuroradiology, Toronto Western Hospital, University of Toronto, Toronto, Ontario, Canada

${ }^{4}$ Department of Neurological Surgery, Miami Miller School of Medicine, University of Miami Hospital, Miami, Florida, USA ${ }^{5}$ Department of Radiology, University of Miami Hospital, Miami Miller School of Medicine, Miami, Florida, USA ${ }^{6}$ Evidence-based Practice Center, Mayo Clinic, Rochester, Minnesota, USA ${ }^{7}$ Department of Neurosurgery, State University of New York at Stony Brook, Stony Brook University Medical Center, Stony Brook, New York, USA

${ }^{8}$ Department of Neuroradiology, Toronto Western Hospital, University of Toronto, Toronto, Ontario, Canada ${ }^{9}$ Department of Diagnostic Imaging, University of Calgary, Calgary, Alberta, Canada
\end{abstract}

\section{Correspondence to} Dr Waleed Brinjikji, Department of Radiology, Mayo Clini, Rochester, MN 55905 Minnesota; brinjikji.waleed@ gmail.com

Received 5 May 2017 Revised 2 June 2017

Accepted 9 June 2017 Published Online First 28 July 2017

\begin{abstract}
Background and purpose Flow arrest with balloon guide catheters (BGCs) is becoming increasingly recognized as critical to optimizing patient outcomes for mechanical thrombectomy. We performed a systematic review and meta-analysis of the literature for studies that compared angiographic and clinical outcomes for patients who underwent mechanical thrombectomy with and without BGCs.
\end{abstract}

Materials and methods In April 2017 a literature search on BGC and mechanical thrombectomy for stroke was performed. All studies included patients treated with and without BGCs using modern techniques (ie, stent retrievers). Using random effects meta-analysis, we evaluated the following outcomes: first-pass recanalization, Thrombolysis In Cerebral Infarction ( $\mathrm{TICl}$ ) 3 recanalization, $\mathrm{TICl} 2 \mathrm{~b} / 3$ recanalization, favorable outcome (modified Rankin Scale (mRS) 0-2), mortality, and mean number of passes and procedure time.

Results Five non-randomized studies of 2022 patients were included (1083 BGC group and 939 non-BGC group). Compared with the non-BGC group, patients treated with BGCs had higher odds of first-pass recanalization (OR 2.05, 95\% Cl 1.65 to 2.55$), \mathrm{TICl} 3$ (OR 2.13,95\% Cl 1.43 to 3.17), $\mathrm{TICl} 2 \mathrm{~b} / 3$ (OR 1.54, $95 \% \mathrm{Cl} 1.21$ to 1.97 ), and mRS 0-2 (OR $1.84,95 \% \mathrm{Cl}$ 1.52 to 2.22). BGC-treated patients also had lower odds of mortality (OR $0.52,95 \% \mathrm{Cl} 0.37$ to 0.73 ) compared with non-BGC patients. The mean number of passes was significantly lower for BGC-treated patients (weighted mean difference $-0.34,95 \% \mathrm{Cl}-0.47$ to -0.22 ). Mean procedure time was also significantly shorter for BGCtreated patients (weighted mean difference $-7.7 \mathrm{~min}$, 95\% Cl-9.0to -6.4).

Conclusions Non-randomized studies suggest that BGC use during mechanical thrombectomy for acute ischemic stroke is associated with superior clinical and angiographic outcomes. Further randomized trials are needed to confirm the results of this study.

\section{INTRODUCTION}

With mechanical thrombectomy now representing standard of care for treatment of acute ischemic stroke secondary to large vessel occlusion (LVO), identifying adjunctive techniques that result in improved outcomes for patients with LVO has become an issue of increasing importance. ${ }^{1-6}$ Over the past several years there has been growing interest in the use of flow arrest with a balloon guide catheter (BGC) as a technique to improve both angiographic and clinical outcomes. ${ }^{7-9}$ A number of benchtop studies have demonstrated that flow arrest during the clot retrieval process results in less clot fragmentation, lower rates of distal emboli, and more effective revascularization. ${ }^{710}$ These findings are further supported by clinical studies which have demonstrated that BGCs are associated with higher revascularization rates and improved rates of good neurological outcome. ${ }^{89}$

Despite the preponderance of data in favor of BGC use, most large registries suggest that only about $50 \%$ of stroke interventions are performed with a BGC, even in the most recent clinical trials. Due to the continuing debate regarding the use of flow arrest during mechanical thrombectomy, we performed a systematic review and meta-analysis of studies comparing outcomes of patients undergoing mechanical thrombectomy who did and did not undergo flow arrest with a BGC during the stroke intervention procedure.

\section{METHODS}

\section{Literature search}

Our study adheres to PRISMA guidelines. In order to identify comparative studies on the use of BGCs during endovascular treatment of acute ischemic stroke, three databases were searched from January 2010 to April 2017: Ovid MEDLINE, Ovid EMBASE, and the Web of Science. The initial search terms were balloon guide catheter, flow arrest, stroke, and endovascular, thrombectomy, and embolectomy. We also searched references from multiple articles to find any additional studies on BGCs and outcomes of endovascular treatment of acute ischemic stroke not found in the initial literature search and contacted experts in the field for any additional studies that provided data on the use of BGCs and outcomes of stroke intervention.

Identified studies from the literature search were then further evaluated for inclusion in the meta-analysis. Inclusion criteria were: (1) studies comparing outcomes of two groups (BGC and non-BGC); and (2) studies reporting separate angiographic and/or clinical outcomes for BGC and non-BGC groups. Exclusion criteria were the following: (1) case reports; (2) studies not separating outcomes by BGC use; and (3) non-comparative studies (ie, studies with only one group). 
Risk of bias assessment

Risk of bias assessment of the studies was performed using the modified Newcastle Ottawa Scale. This is a tool used for assessing the quality of non-randomized studies included in systematic reviews and/or meta-analyses. Each study is judged on eight items categorized into three groups: (1) selection of the study groups; (2) comparability of the study groups; and (3) ascertainment of the outcome of interest. ${ }^{11}$ For our topic of interest, the factors that would make a study have a low risk of bias include (1) well-defined selection criteria; (2) similar baseline NIH Stroke Scale (NIHSS) score, occlusion location and treatment paradigms between groups; and (3) independent assessment of neurological and angiographic outcomes. The quality of evidence was assessed using the GRADE approach (Grading of Recommendations, Assessment, Development and Evaluation).

\section{Outcome variables}

For the purposes of this study, patients were divided into two groups: BGC and non-BGC. The following outcomes were studied: good functional outcome, defined as a modified Rankin score (mRS) of $\leq 2$ at 90 days following endovascular treatment, mortality, successful recanalization/angiographic outcome defined as Thrombolysis In Cerebral Infarction (TICI) $2 b / 3$, TICI 3, first pass recanalization rates, mean number of passes, and procedure time.

\section{Statistical analysis}

From each study we extracted a $2 \times 2$ table for binary outcomes and the mean, group sample size and a measure of variability for continuous outcomes. Random effects meta-analysis was used for pooling across studies. ${ }^{12}$ The $\mathrm{I}^{2}$ statistic was used to express the proportion of heterogeneity that is not attributable to chance. ${ }^{13}$ Meta-analysis results were expressed as OR for binary outcomes and weighted mean difference (WMD) for continuous outcomes with respective $95 \%$ CIs. Due to the small number of studies, we performed a sensitivity analysis by analyzing the data using fixed-effects model as well. We explored the impact of publication bias by constructing funnel plots and testing their symmetry. Meta-analysis and meta-regression were conducted using STATA Version 14 (StataCorp LP, College Station, Texas, USA).

\section{RESULTS}

\section{Literature search}

A total of 752 articles were found from the literature search. Of these, 744 were excluded after reading the abstracts alone as they were not found to be relevant to our study. Of the remaining eight articles and conference abstracts, four were excluded because they did not include a control group of non-BGC treated patients. One additional study/conference abstract was found when contacting experts in the field. In total, five studies with 2022 patients were included (1083 BGC group and 939 non-BGC group; figure 1). ${ }^{8914-16}$ All patients were treated with stent retriever technology. The largest study had 880 patients and the smallest study had 87 patients.

All patients in the included studies were treated with stent retrievers. All but one study only included patients with anterior circulation strokes. Posterior circulation strokes represented $0.6 \%(7 / 1083)$ of the BGC group and 3.0\% (28/939) of the non-BGC group. No study demonstrated a difference in baseline NIHSS between groups. In two studies there were important differences in baseline demographics and comorbidities between groups. Four studies were post hoc analyses
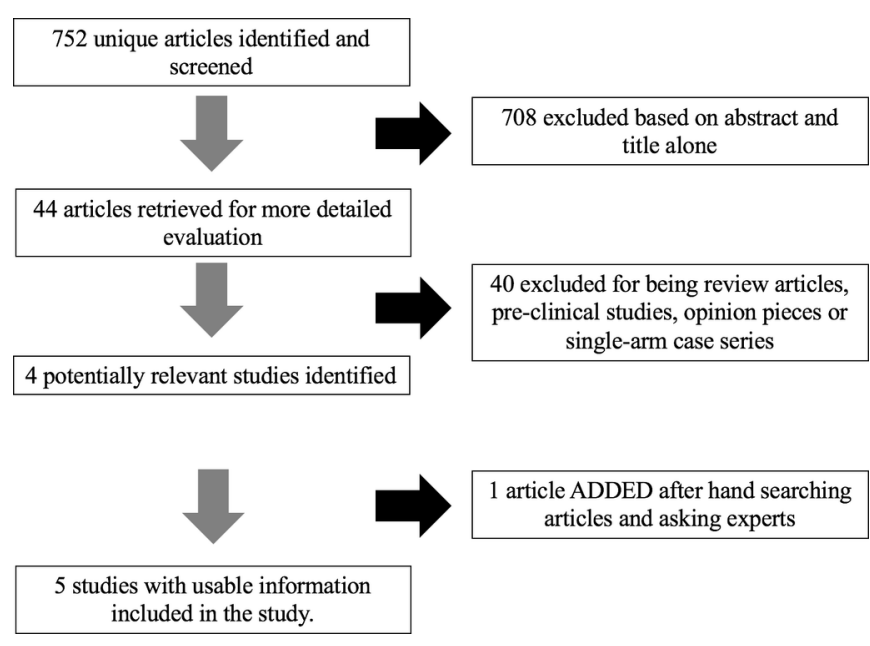

Figure 1 PRISMA flow diagram.

of multicenter prospective registries or clinical trials and two included core laboratory analysis of outcomes. Risk of bias was moderate in four studies and high in one study. These data are summarized in table 1.

\section{Clinical outcomes}

BGC-treated patients had mRS 0-2 rates of 59.7\% (572/958) compared with 43.8\% (369/842) for non-BGC-treated patients (OR 1.84, 95\% CI 1.52 to 2.22, p $<0.01$; figure 2). Mortality rates were significantly lower in BGC-treated patients $(62 / 453$, $13.7 \%)$ than in non-BGC-treated patients $(116 / 467,24.8 \%)$ (OR $0.52,95 \%$ CI 0.37 to $0.73, \mathrm{p}<0.01$ ). These data are summarized in table 2 .

\section{Angiographic and procedural outcomes}

The overall rate of first-pass recanalization was $63.1 \%$ (497/788) for the BGC group compared with 45.2\% (280/619) for the non-BGC group (OR 2.05, 95\% CI 1.65 to $2.55, \mathrm{p}<0.01$ ). The rate of TICI 3 was $57.9 \%(114 / 197)$ in the BGC group and $38.2 \%(87 / 228)$ for the non-BGC group (OR 2.13, 95\% CI 1.43 to $3.17, \mathrm{p}<0.01)$ and the rate of TICI $2 \mathrm{~b} / 3$ was $78.9 \%(817 / 1035)$ for the BGC group and $67.0 \%(603 / 900)$ for the non-BGC group (OR 1.54, 95\% CI 1.21 to $1.97, \mathrm{p}<0.01$; figure 3 ). These data are summarized in table 2 .

The mean number of passes was 1.7 (95\% CI 1.6 to 1.8) for BGC-treated patients and 2.0 (95\% CI 1.9 to 2.2) for non-BGCtreated patients (WMD $-0.34,95 \%$ CI -0.47 to -0.22 , $\mathrm{p}<0.01)$. Mean procedure time was $70.5 \mathrm{~min}(95 \%$ CI 37.5 to 103.5$)$ for the BGC group compared with $90.9 \mathrm{~min}(95 \%$ CI 47.1 to 134.7 ) for the non-BGC group (WMD -7.7 , $95 \% \mathrm{CI}-9.0$ to $-6.4, \mathrm{p}<0.01)$. These data are summarized in table 3 .

\section{Heterogeneity, publication bias and quality of evidence}

$\mathrm{I}^{2}$ values were $67 \%$ for TICI $2 \mathrm{~b} / 3,66 \%$ for mean number of passes, and $98 \%$ for mean procedure time. $\mathrm{I}^{2}$ values were $10 \%$ for first-pass recanalization, $0 \%$ for TICI 3, 0\% for mRS $0-2$, and $50 \%$ for mortality. The $\mathrm{p}$ value for publication bias using Egger's regression was 0.49 (figure 4), suggesting no bias, although this is not reliable due to the small number of studies. The quality of evidence supporting all outcomes was low due to the non-randomized nature of the included studies and heterogeneity. 


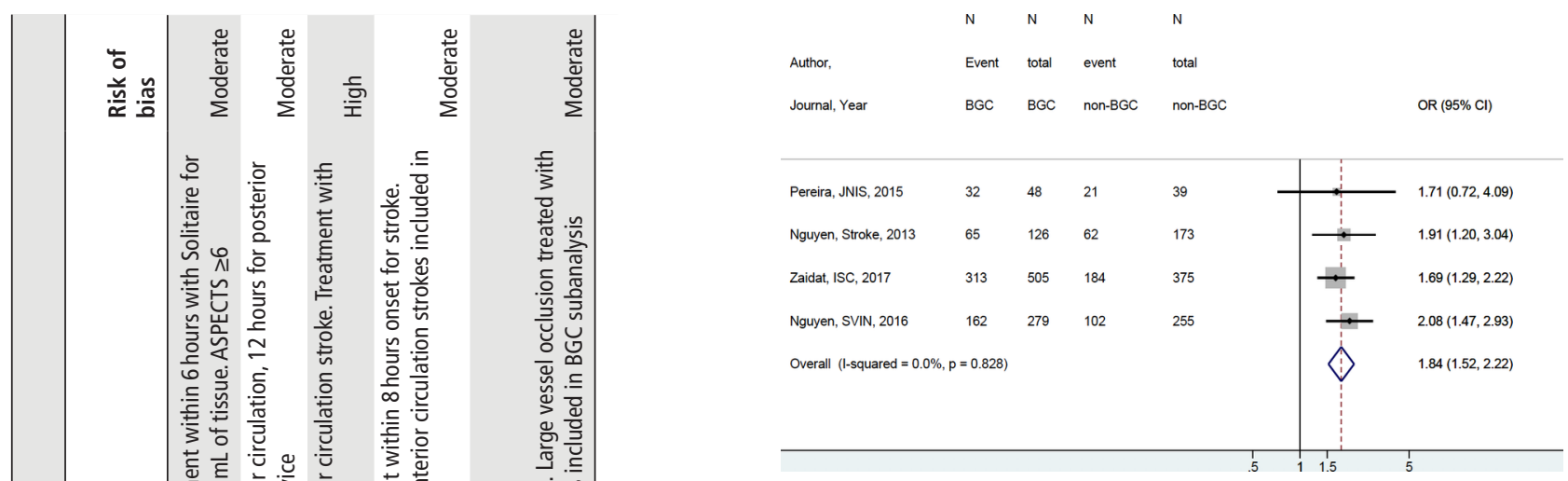

Figure 2 Forest plot for modified Rankin Scale (mRS) 0-2.

\section{Sensitivity analysis}

Sensitivity analysis using a fixed effects model showed no significant change in meta-analysis results when compared with the random effects model. Sensitivity analysis removing the largest study (Zaidat et al, STRATIS subgroup analysis) ${ }^{16} 17$ from our primary outcome of mRS 0-2 showed similar results to our overall analysis-namely, BGC use was associated with a significantly higher odds of good neurological outcome than non-BGC use with no heterogeneity (OR 2.0, 95\% CI 1.52 to $\left.2.59, \mathrm{I}^{2}=0 \%\right)$. When excluding patients with posterior circulation stroke, BGC use was associated with a significantly higher odds of good neurological outcome than non-BGC use with no heterogeneity (OR $1.85,95 \% \mathrm{CI} 1.52$ to $2.24, \mathrm{I}^{2}=0 \%$ ).

\section{DISCUSSION}

Our systematic review and meta-analysis of five studies including over 2000 patients found that the use of BGC during stroke intervention with a stent retriever is associated with significantly improved clinical, angiographic, and procedural outcomes. BGC-treated patients had significantly higher rates of good neurological outcome and nearly half the mortality rate of non-BGC-treated patients. While there was a $12 \%$ difference in TICI $2 \mathrm{~b} / 3$ rates, there was a $20 \%$ difference in TICI 3 , indicating that BGC use resulted in less clot fragmentation and distal embolization. The fact that first-pass recanalization rates were over $60 \%$ was probably the key factor in the 20 min reduction in procedure time associated with BGC use. These findings are important as they suggest that BGC use may be a key factor in improving outcomes of patients receiving endovascular treatment for LVO.

A number of preclinical benchtop studies have highlighted the mechanism by which BGCs confer benefit during stroke

\begin{tabular}{|c|c|c|c|c|c|c|}
\hline & $\begin{array}{l}\text { Number of } \\
\text { studies }\end{array}$ & BGC & No $B G C$ & $\begin{array}{l}\text { OR } \\
(95 \% \mathrm{Cl})\end{array}$ & $\mathrm{p}$ Value & $\mathrm{I}^{2}$ \\
\hline $\begin{array}{l}\text { First-pass } \\
\text { recanalization }\end{array}$ & 3 & $63.1 \%$ & $45.2 \%$ & $\begin{array}{l}2.05(1.65 \\
\text { to } 2.55)\end{array}$ & $<0.01$ & $10 \%$ \\
\hline $\mathrm{TICl} 3$ & 2 & $57.9 \%$ & $38.2 \%$ & $\begin{array}{l}2.13(1.43 \\
\text { to } 3.17)\end{array}$ & $<0.01$ & $0 \%$ \\
\hline $\mathrm{TICl} 2 \mathrm{~b} / 3$ & 4 & $78.9 \%$ & $67.0 \%$ & $\begin{array}{l}1.54(1.21 \\
\text { to } 1.97)\end{array}$ & $<0.01$ & $67 \%$ \\
\hline mRS 0-2 & 4 & $59.7 \%$ & $43.8 \%$ & $\begin{array}{l}1.84(1.52 \\
\text { to } 2.22)\end{array}$ & $<0.01$ & $0 \%$ \\
\hline Mortality & 3 & $13.7 \%$ & $24.8 \%$ & $\begin{array}{l}0.52(0.37 \\
\text { to } 0.73)\end{array}$ & $<0.01$ & $50 \%$ \\
\hline
\end{tabular}




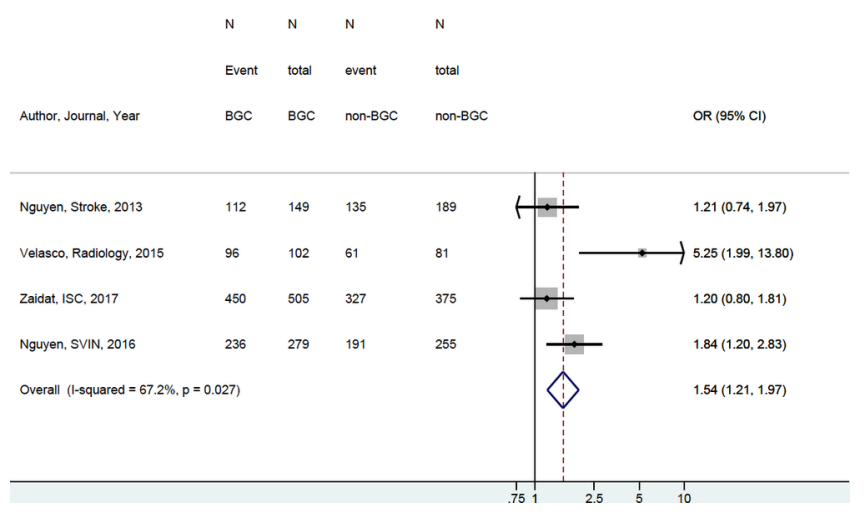

Figure 3 Forest plot for Thrombolysis In Cerebral Infarction (TICI) $2 b / 3$.

intervention. ${ }^{710}$ In a study using a silicon LVO model of stroke, Chueh et al found that the use of a BGC resulted in a significant reduction in large clot fragments as well as improved recanalization. ${ }^{7}$ Interestingly, the reduction in distal emboli was 18 -fold higher than controls when the clot was a fragile clot and twofold higher than controls when the clot was an elastic clot. ${ }^{7}$ These findings are corroborated by the findings of our study which showed that patients who were treated with a BGC had a nearly $20 \%$ higher rate of TICI 3 than those treated without a BGC.

Over the past several years a number of studies have demonstrated the clinical benefits of BGCs in the treatment of patients with acute ischemic stroke. In fact, evidence in favor of BGC use has been so compelling that it was a mandatory part of the stroke intervention procedure in the EXTEND-IA study and STAR Trial. ${ }^{2}$ In a post hoc analysis of the NASA registry, Nguyen et al found that BGC use was associated with significantly higher rates of TICI 3 and good clinical outcome and significantly shorter procedure times. ${ }^{8}$ In their study, BGC use was independently associated with a good clinical outcome on multivariate analysis. ${ }^{8}$ In a retrospective study of two institutions, Velasco et al found that the use of a BGC was associated with higher rates of successful revascularization and a nearly twofold higher rate of one-pass thrombectomy. Furthermore, procedure time was 50\% shorter in the BGC group than in the non-BGC group. ${ }^{9}$ In the ESCAPE trial, BGC use was associated with a $21 \%$ reduction in procedure time $(8 \mathrm{~min}) .{ }^{17}$ Findings from our meta-analysis provide further compelling evidence in support of BGC use with significant reductions in procedure time and improvements in revascularization rates and clinical outcomes.

BGCs are not without their limitations. When using a BGC, one must use an $8 \mathrm{Fr}$ or $9 \mathrm{Fr}$ groin sheath, which is larger than the typical $6 \mathrm{Fr}$ access needed for performing direct aspiration. It is important to point out that recently published studies have very low groin complication rates with $8 \mathrm{Fr}$ and $9 \mathrm{Fr}$ sheaths however. ${ }^{18}$ There are issues with stability when using a BGC, particularly in difficult arches. In one of the studies included in our analysis, patients treated with BGCs were younger than those treated without a BGC, suggesting that there was a bias to treating patients with more tortuous arches without the use of a BGC. ${ }^{14}$

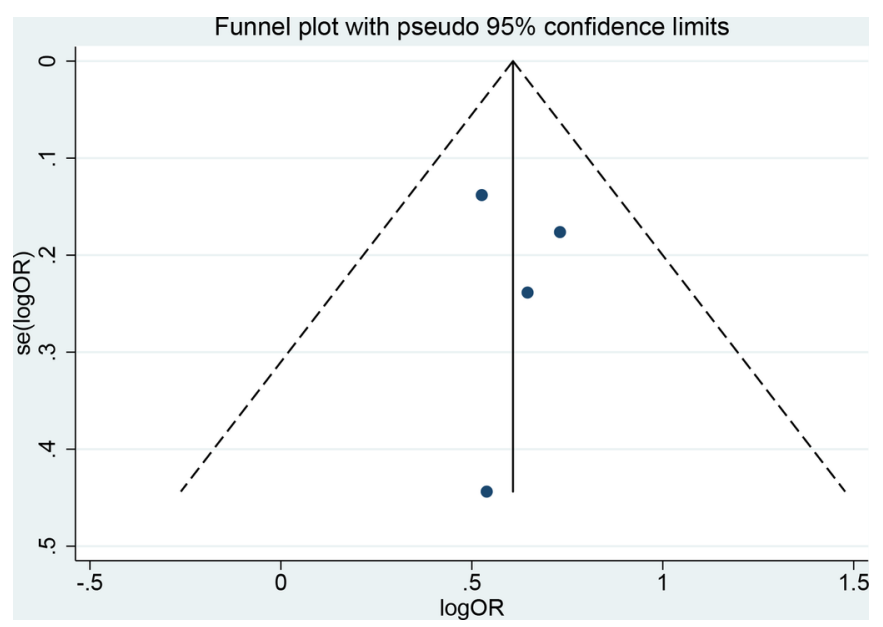

Figure 4 Funnel plot for publication bias.

\section{Limitations}

Our study has limitations. All of the included studies were post hoc analyses of registries or clinical trials or small retrospective experiences of a few centers. Because of this, there is a possibility for selection bias. Three of the included studies were conference abstracts which have not yet been published as full-text articles. However, the Institute of Medicine Standards of Systematic Reviews recommends searching trial registries, conference abstracts, regulatory documents, and other sources of unpublished information for the purposes of systematic reviews. ${ }^{19}$ Another limitation is the fact that multiple types of stent retrievers and BGCs were used in this study which introduces some heterogeneity. Due to the small number of studies we were unable to study outcomes by occlusion location (ie, M1, ICA bifurcation) and other important baseline characteristics. Despite the high likelihood of bias and confounding, the meta-analytic estimates presented are the best available evidence and decisions have to be based on the available evidence. Using GRADE, the quality of evidence is low, which reflects the presence of bias.

We were unable to compare outcomes of patients treated using direct aspiration without BGCs with those of patients treated with BGCs with stent retriever or aspiration. Data on the use of distal access catheters, aspiration techniques, and pinning techniques were not available in the included studies. This is particularly important as some suggest that the continuous aspiration that is performed when using distal access catheters may reduce clot fragmentation and distal emboli. However, benchtop studies demonstrate that flow reversal is significantly improved when using a BGC compared with a conventional guide catheter. ${ }^{7}$ It is important to point out that continuous aspiration does not result in flow arrest as, once the aspiration catheter gets clogged by the very first part of the clot, the remainder of the clot is fully exposed to the strong arterial flow in the carotid and middle cerebral artery. Data from the Interest of Direct Aspiration First Pass Technique (ADAPT) for Thrombectomy Revascularization of Large Vessel Occlusion in Acute Ischemic Stroke (ASTER) trial

Table 3 Continuous outcomes

\begin{tabular}{lllllll}
\hline & Number of studies & BGC & No BGC & Mean difference (95\% Cl) & p Value & I $^{2}$ \\
\hline Mean number of passes (SD) & 4 & $1.7(1.6$ to 1.8$)$ & $2.0(1.9$ to 2.2$)$ & $-0.34(-0.47$ to -0.22$)$ & $<0.01$ & $66 \%$ \\
\hline Mean procedure time, $\min$ (SD) & 4 & $70.5(37.5$ to 103.5$)$ & $90.9(47.1$ to 134.7$)$ & $-7.7(-9.0$ to -6.4$)$ & $<0.01$ & $98 \%$ \\
\hline
\end{tabular}


suggest that there were no statistically significant differences in revascularization rates when performing the ADAPT technique compared with using a stent retriever. ${ }^{20}$ However, it is unclear at this time whether BGCs were used in this trial. Lastly, use of a BGC does not necessarily mean that the actual technique of using a BGC (appropriate positioning, sufficient inflation, aspiration, etc) was appropriate or effective. Thus, is possible that many patients in the BGC group did not actually achieve flow arrest.

It is interesting to point out that procedure times in the BGC and non-BGC groups were still high when compared with rates that have been previously reported in the literature (70 min and $90 \mathrm{~min}$, respectively). This is despite the fact that first-pass recanalization rates were as high as $60 \%$ in the BGC group. There is an apparent incongruence which is difficult to explain based on the data presented in the included articles.

\section{CONCLUSIONS}

Data from our meta-analysis suggest that BGC use is associated with significant improvement in procedural, angiographic, and clinical outcomes. Further rigorously conducted studies are needed to confirm these results. Ultimately, a randomized controlled trial comparing the efficacy of BGC versus no-BGC may be needed to determine if BGC use should be standard of care.

\section{Twitter@wbrinjikji}

Contributors WB, RMS, DF, DFK, VMP, and MG made (1) substantial contributions to the conception or design of the work or the acquisition, analysis or interpretation of data for the work; (2) drafting of the work or revising it critically for important intellectual content; (3) final approval of the version to be published; and (4) are in agreement to be accountable for all aspects of the work in ensuring that questions related to the accuracy or integrity of any part of the work are appropriately investigated and resolved.

Funding WB is CEO of Marblehead Medical LLC and has patents pending in balloon catheter technologies. DFK is President of Marblehead Medical LLC and has patents pending in balloon catheter technologies. There are no other relevant disclosures.

Provenance and peer review Not commissioned; externally peer reviewed.

Data sharing statement Data can be made available by contacting the corresponding author via email.

(c) Article author(s) (or their employer(s) unless otherwise stated in the text of the article) 2018. All rights reserved. No commercial use is permitted unless otherwise expressly granted.

\section{REFERENCES}

1 Campbell BC, Hill MD, Rubiera M, et al. Safety and efficacy of Solitaire stent thrombectomy: individual patient data meta-analysis of randomized trials. Stroke 2016;47:798-806.
2 Campbell BC, Mitchell PJ, Kleinig TJ, et al. Endovascular therapy for ischemic stroke with perfusion-imaging selection. N Eng/ J Med 2015;372:1009-18.

3 Goyal M, Demchuk AM, Menon BK, et al. Randomized assessment of rapid endovascular treatment of ischemic stroke. N Engl J Med 2015;372:1019-30.

4 Jovin TG, Chamorro A, Cobo E, et al. Thrombectomy within 8 hours after symptom onset in ischemic stroke. N Engl J Med 2015;372:2296-306.

5 Saver JL, Goyal M, Bonafe A, et al. Stent-retriever thrombectomy after intravenous t-PA vs. t-PA alone in stroke. N Engl J Med 2015;372:2285-95.

6 Berkhemer $\mathrm{OA}$, Fransen PS, Beumer $\mathrm{D}$, et al. A randomized trial of intraarterial treatment for acute ischemic stroke. N Eng/ J Med 2015;372:11-20.

7 Chueh JY, Kühn AL, Puri AS, et al. Reduction in distal emboli with proximal flow control during mechanical thrombectomy: a quantitative in vitro study. Stroke 2013:44:1396-401.

8 Nguyen TN, Malisch T, Castonguay AC, et al. Balloon guide catheter improves revascularization and clinical outcomes with the Solitaire device: analysis of the North American Solitaire Acute Stroke Registry. Stroke 2014;45:141-5.

9 Velasco A, Buerke B, Stracke CP, et al. Comparison of a balloon guide catheter and a non-balloon guide catheter for mechanical thrombectomy. Radiology 2016;280:169-76.

10 Mokin M, Setlur Nagesh SV, lonita CN, et al. Stent retriever thrombectomy with the Cover accessory device versus proximal protection with a balloon guide catheter: in vitro stroke model comparison. J Neurointerv Surg 2016;8:413-7.

11 Deeks JJ, Dinnes J, D'Amico R, et al. Evaluating non-randomised intervention studies. Health Technol Assess 2003;7:iii-x, 1-173.

12 DerSimonian R, Laird N. Meta-analysis in clinical trials. Control Clin Trials 1986:7:177-88.

13 Higgins JP, Thompson SG, Deeks JJ, et al. Measuring inconsistency in meta-analyses. BMJ 2003;327:557-60.

14 Nguyen T, Castonguay AC, Nogueira RN, et al. Balloon guide catheter improved clinical outcomes, revascularization, and decreased mortality in Trevo thrombectomy. Analysis of the TREVO Stent Retriever acute stroke (TRACK) Registry. Presented at the Society of Vascular Interventional Neurology Conference.http://www.svin.org/ files/Annual\%20Meeting/2015/Platform\%20Session\%20ll\%20TOC\%20\%26\% 20Presentations.pdf

15 Zaidat 0, Froehler MT, Aziz-Sulta MA, et al. LBP2 influence of balloon, conventional or distal catheters on angiographic and clinical outcomes in the Stratis Registry. Stroke 2017:48. LBP2.

16 Zaidat O, Liebeskind D, Jahan R, et al. Influence of balloon, conventional, or distal catheters on angiographic and technical outcomes in STRATIS. J Neurointerv Surg 2016;8:A3.2-A4

17 Menon BK, Sajobi TT, Zhang Y, et al. Analysis of orkflow and ime to treatment on thrombectomy outcome in the endovascular treatment for small core and proximal occlusion ischemic stroke (ESCAPE) randomized, controlled trial. Circulation 2016;133:2279-86.

18 Shah VA, Martin CO, Hawkins AM, et al. Groin complications in endovascular mechanical thrombectomy for acute ischemic stroke: a 10-year single center experience. J Neurointerv Surg 2016;8:568-70.

19 Eden J, Levit L, Berg A, Morton S, eds. Finding what works in health care: Standards for systematic reviews. Washington 2011.

20 Lapergue B, Piotin M. Aster trial. Contact aspiration versus stent retriever front line for recanalisation in acute cerebral infarction.. Stroke 2017;48:LB2.

21 Pereira V, Siddiqui A, Jovin T, et al. Role of balloon guiding catheter in mechanical thrombectomy using stentretrivers subgroup analysis of swift prime: Abstract P-016. J Neurointerv Surg 2015;7:A30. 